Tropical Journal of Pharmaceutical Research February 2013; 12 (1): 1-6

ISSN: $1596-5996$ (print); 1596-9827 (electronic)

(c) Pharmacotherapy Group, Faculty of Pharmacy, University of Benin, Benin City, 300001 Nigeria.

All rights reserved.

Available online at http://www.tjpr.org

Original Research Article

http://dx.doi.org/10.4314/tjpr.v12i1.1

\title{
Preparation, Characterisation and In Vivo Evaluation of Silybin Nanoparticles for the Treatment of Liver Fibrosis
}

\author{
Chandrasekhar R Bonepally ${ }^{1}$, Sai Jyothsna Gandey ${ }^{1,}$ Krishnaveni Bommineni ${ }^{1}$, \\ Krishna Mohan Gottumukkala ${ }^{2}$ and Jithan Aukunuru ${ }^{3^{*}}$ \\ ${ }^{1}$ Vaagdevi College of Pharmacy, Warangal, AP, ${ }^{2}$ Department of Pharmaceutical Sciences, JNTU, Hyderabad, AP, ${ }^{3}$ Mother \\ Teresa College of Pharmacy, Hyderabad, AP, India
}

*For correspondence: E-mail: aukunjv@gmail.com

Received: 17 November 2011

Revised accepted: 25 November 2012

\begin{abstract}
Purpose: To formulate and characterize nanoparticles containing silybin, and evaluate their activity against carbon tetrachloride $\left(\mathrm{CCl}_{4}\right)$-induced liver toxicity.

Methods: Silybin nanoparticles were formulated by o/w emulsion solvent evaporation technique using

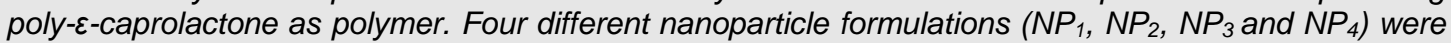
prepared by varying the drug/polymer ratio. The particles were characterized for particle size, drug content and in vitro drug release. The pharmacokinetics and pharmacodynamics of the silybin formulations in male Wistar rats were evaluated following i.v. administration, using silybin solution as reference. The hepatoprotective activity of the formulations was also determined in a $\mathrm{CCl}_{4}$-treated rat model.

Results: Silybin nanoparticles were successfully prepared using o/w emulsion solvent evaporation technique. The nanoparticles sustained the release of the drug both in vitro and in vivo for up to 10 days and offered better pharmacokinetic properties than the free drug itself. Intravenous nanoparticulate administration reversed serum liver enzyme levels by $95 \%$ compared to only $50 \%$ for the drug solution. Conclusion: The developed silybin nanoparticles showed superior pharmacokinetic properties and hepatoprotective activity to silybin solution.
\end{abstract}

Keywords: Silybin, Nanoparticles, Pharmacokinetics, Pharmacodynamics, Hepatoprotective activity.

Tropical Journal of Pharmaceutical Research is indexed by Science Citation Index (SciSearch), Scopus, International Pharmaceutical Abstract, Chemical Abstracts, Embase, Index Copernicus, EBSCO, African Index Medicus, JournalSeek, Journal Citation Reports/Science Edition, Directory of Open Access Journals (DOAJ), African Journal Online, Bioline International, Open-J-Gate and Pharmacy Abstracts

\section{INTRODUCTION}

Non-alcoholic and alcoholic fatty liver, chronic hepatitis and hepatic carcinoma all of which causes liver fibrosis that results in irreversible cirrhosis ultimately death in human beings $[1,2]$. Liver fibrosis is the accumulation of extracellular matrix or scar in the liver. There is no standard treatment for liver fibrosis [3]. Thus there is a need to find effective treatment for fibrosis. The ideal antifibrotic therapy would be one that is liver-specific, well tolerated when administered for prolonged periods of time, effective in attenuating excessive collagen deposition without affecting normal ECM synthesis, effectively delivered and nontoxic to other organs[4].

Silybin is one of the oldest drugs for the treatment of liver fibrosis and cirrhosis [5]. Although it is considered to be ideal for the 
treatment of liver fibrosis, delivery to the liver still needs improvement. The effectiveness of oral silybin as a hepatoprotective agent has discounted by its poor solubility, low bioavailability and low half-life [6]. Sylibin needs to be administered daily to achieve its effects. Nanosized carriers encapsulating sylibinin with size less than $6 \mu \mathrm{m}$ can be taken up passively into Kupffer cells in the liver and can result in increased drug concentration in the liver after intravenous administration, thus increasing therapeutic efficacy. They can result in sustained systemic release of sylibin for more than a week, depending on various factors, after forming a depot in the Kupffer cells. Thus, repeated daily administration for sylibin can be avoided. Further, oral bioavailability problems with sylibin can be avoided since bioavailability is $100 \%$ after intravenous administration.

Oxidative stress in Kupffer cells is known to initiate the formation of liver fibrosis in many diseases and thus antioxidant sylibin levels in these cells, if enhanced, can tremendously improve therapy with sylibin. Thus, with this type of formulation, sustained parenteral release, improvement in bioavailability as well as enhancement of biochemical protection can be achieved. Together, these mechanisms lead to increase in effectiveness of therapy. Thus, the objective of this study was to prepare biodegradable nanoparticles of silybin, and evaluate their sustained release characteristics, liver targetability and liver protection following intravenous administration.

\section{EXPERIMENTAL}

Silybin and Poly- $\varepsilon$-caprolactone (mol wt,, 14,000) were procured from Sigma-Aldrich, Germany. Polyvinyl alcohol (PVA, cold-water soluble) was procured from Qualikems Fine Chemicals Pvt Ltd, New Delhi. Dichloromethane was procured from SD Fine Chemicals Ltd, Mumbai, India. All other reagents were of analytical grade. A probe sonicator (Homogenizer 150 VT), used to prepare the nanoparticles, was procured from M/S Biologics, Inc USA. A zeta sizer (3000 HAS (Malvern Instruments, Malvern, UK) was used to measure the particle size. (HPLC (Waters, USA) was used to analyze plasma samples while UVVisible spectrophotometer (Shimadzu UV-1800, Japan) was used to analyze drug loading and drug release samples. A magnetic stirrer (Remi Industries, Mumbai, India) was used to facilitate evaporation of dichloromethane while an ultracentrifuge (Remi, Mumbai) was employed to recover the nanoparticles after preparation. Male Wistar rats weighing 150 - $180 \mathrm{~g}$ were purchased from Mahaveer Enterprises, Hyderabad., India

\section{Preparation of silybin nanoparticles}

Emulsion (o/w) solvent evaporation method was employed in the preparation of silybin nanoparticles using poly- $\varepsilon$-caprolactone as the polymer. Four different nanoparticle formulations $\mathrm{NP}_{1}, \mathrm{NP}_{2}, \mathrm{NP}_{3}$ and $\mathrm{NP}_{4}$ containing drug:polymer in the ratio of $1: 1,1: 2,1: 3$ and $1: 4$, respectively, were prepared. For the preparation, silybin (100 $\mathrm{mg})$ and polycaprolactone (100, 200, 300 or 400 $\mathrm{mg}$ ) was dissolved in $15 \mathrm{ml}$ of dichloromethane by vortexing. The mixture (organic phase) was added drop-wise to $50 \mathrm{ml}$ of $2 \%$ PVA solution under probe sonication at $40 \mathrm{w}$ for $12 \mathrm{~min}$ to obtain a w/o emulsion. This emulsion was placed on a magnetic stirrer to ensure complete evaporation of dichloromethane, leaving nanoparticle suspension. The suspension was centrifuged at $10,000 \mathrm{rpm}$ for $20 \mathrm{~min}$, resulting in the formation of a pellet at the bottom of the tube. This pellet was washed with phosphate buffered saline (PBS), re-suspended and again centrifuged. The pellet was collected and allowed to dry completely. The powdered particles were collected, weighed and used for further evaluation.

\section{Determination of particle size and charge}

The nanoparticles were evaluated for their particle size, polydispersity index of size distribution and surface charge potential, by photon correlation spectroscopy (PCS) using Zetasizer 3000 HAS (Malvern Instruments, Malvern, UK). The formulations were diluted $1: 1000$ with the aqueous phase of the formulation to obtain suitable kilo-counts per second (kcps). Analysis was performed at $25^{\circ} \mathrm{C}$ with an angle of detection of $90^{\circ}$. Each determination was made in triplicate.

\section{Evaluation of encapsulation efficiency}

Encapsulation efficiency (EE) was calculated by estimating the amount of unentrapped drug. This was found by measuring the absorbance of the drug in supernatant, which was obtained after centrifugation of the nanoparticle suspension and then applying Eq 1.

$\operatorname{EE~}(\%)=100\left(W_{1}-W_{2}\right) / W_{1}$

The value obtained from $\mathrm{Eq} 1$ was compared with entrapped drug. For this determination, an accurately weighed amount of nanoparticles was taken in a test tube, dissolved in dichloromethane (DCM) and the solvent allowed 
to evaporate completely. An aliquot of methanol $(10 \mathrm{ml})$ was added to the test tube which dissolved only the drug. The absorbance of this solution was measured and the amount of drug encapsulated calculated.

\section{In vitro release study}

The in vitro release study was performed in a diffusion cell set-up across a dialysis membrane. An inverted cylindrical test tube cut to a height of $8 \mathrm{~cm}$ was used as a donor compartment. The receiver compartment consisted of $100 \mathrm{ml}$ of phosphate buffer $\left(\mathrm{pH} 7.4,37^{\circ} \mathrm{C}\right)$ in a beaker placed over a water bath. A dialysis membrane which was pre-soaked in warm water for $30 \mathrm{~min}$ was placed at the lower end of the cylindrical setup and the membrane separated the donor compartment from the receiver compartment. Nanoparticles containing $20 \mathrm{mg}$ of drug was suspended into $5 \mathrm{ml}$ of $\mathrm{pH} 7.4$ buffer and placed in the donor compartment. The system was stirred using a magnetic stirrer and bead. Samples $(5 \mathrm{ml})$ were removed from the receiver compartment and replaced with the same volume of fresh medium immediately. The samples were analyzed spectrophotometrically at $287 \mathrm{~nm}$.

\section{Pharmacokinetic studies}

Male Wister rats (weighing 150 - $180 \mathrm{~g}$ each) were purchased from Mahaveer Enterprises, Hyderabad, India, and were maintained in an airconditioned room at $22 \pm 2{ }^{0} \mathrm{C}$ and relative humidity of $45-55 \%$ in a $12 / 12$ h light/dark cycle. The animals had free access to standard food pellets and water was available ad libitum. All the animal experiments were conducted according to the guidelines of the Committee for the Purpose of Control and Supervision of Experiments on Animals (CPCSEA), Chennai, India [7] and the study protocol was approved by Institutional Animal Ethical Committee of Vaagdevi College of Pharmacy, Warangal, India (ref no. 1047/ac/07/CPCSEA). International guidelines issued by the International Council for Laboratory Animal Science were also followed [8]. These conditions were maintained throughout the duration of the experiment. The study was performed in three groups of six rats each.

Group 1 received silybin solution containing $30 \mathrm{mg} / \mathrm{kg}$ intravenously; Group 2 received $\mathrm{NP}_{1}$ silybin nanoparticles equivalent to $30 \mathrm{mg} / \mathrm{kg}$ of drug suspended in normal saline and injected intravenously while Group 3 received placebo nanoparticles. Blood samples were collected at different time intervals over a period of $24 \mathrm{~h}$. For the nanoparticle formulations samples were also collected days 3, 6 and 9 after administration. Drug levels in the plasma samples were evaluated by HPLC. HPLC standard curve for the drug in plasma was also generated. The UV detection wavelength was $288 \mathrm{~nm}$ while the mobile phase consisted of methanol: water (50:50, v/v). The following pharmacokinetic parameters were determined using WinNonlin pharmacokinetic data analysis software: elimination rate constant (ke), volume of distribution $(\mathrm{Vd})$, elimination half-life $\left(\mathrm{t}_{1 / 2}\right)$, clearance $(\mathrm{CL})$, and area under curve (AUC).

\section{Evaluation of hepatoprotective activity}

Carbon tetrachloride $\left(\mathrm{CCl}_{4}\right)$-induced liver damage model was used in the evaluation of hepatoprotective activity. For this purpose another set of male Wistar rats were divided into five groups each containing 6 rats. Group1 received normal saline ( $1 \mathrm{ml} / \mathrm{rat})$ daily for 9 days and served as normal control. Group 2 received $\mathrm{CCl}_{4}$ (dissolved in 3 times its volume of olive) at a dose of $0.7 \mathrm{ml} / \mathrm{kg}$ intraperitoneally on days 3,6 and 9 and served as toxic control. Group 3 received the drug solution in a dose of $100 \mathrm{mg} / \mathrm{kg}$ intravenously daily for 9 days. Group 4 received silybin nanoparticle suspension equivalent to 100 $\mathrm{mg} / \mathrm{kg}$ of drug intravenously on day 1 while Group5 received placebo nanoparticles. All the groups received $\mathrm{CCl}_{4}$ at days 1, 3, 6 and 9 of the study except normal control.

The animals were anaesthetized on the last day of the study and blood was collected by cardiac puncture. Plasma was separated from the blood samples by centrifugation at $3000 \mathrm{rpm}$ for 15 min. Hepatoprotective activity was quantified by serum glutamate oxaloacetate transaminase (SGOT) and serum glutamate pyruvic transaminase (SGPT) levels in the plasma. Subsequently, their livers were subjected to histopathological examination. First, the rats were sacrificed at the last day of the study, the liver separated carefully and preserved in formalin solution, and liver sections were prepared. ${ }^{[13]}$ The body weight of the rats were also monitored.

\section{Statistical analysis}

The data were expressed as mean \pm standard deviation (SD) and statistical analysis was carried out by one-way ANOVA followed by Student's Newman-Keuls test. The level of significance used was $P<0.05$. The statistical software used was Graph Pad Prism, USA, versions 4 and 5 . 


\section{RESULTS}

Silybin nanoparticles were successfully prepared using poly- $\varepsilon$-caprolactone by $\mathrm{o} / \mathrm{w}$ emulsion solvent evaporation method. The mean particle size of the nanoparticles ranged from $130-430$ $\mathrm{nm}$ (Table 1). Mean particle size increased with increase in polymer concentration. The polydispersity index (PDI) of the nanoparticles was $<0.4$, indicating that the nanoparticles were homogenous in distribution. The zeta potential value indicates long term stability. The encapsulation efficiency of the nanoparticles increased as polymer concentration increased.

\section{In vitro drug release characteristics}

In vitro release data, shown in Fig 1, indicates that all the formulations sustained drug release for over a period of 20 days. A biphasic drug release pattern was found, i.e., burst release followed by sustained release. In the first 6 hours of release, the unencapsulated drug component was released. Subsequently, the encapsulated drug component was gradually released. Drug release from NP1 was higher than from NP2, NP3 and NP4. Log percent cumulative drug released, plotted as a function of log time yielded curves, the slope of is the diffusional release exponent $(\mathrm{n})$. The values of diffusional $\mathrm{n}$ were $0.359,0.374,0.420$ and 0.450 for $\mathrm{NP}_{1}, \mathrm{NP}_{2}, \mathrm{NP}_{3}$ and $\mathrm{NP}_{4}$ respectively, which indicate that drug release from all the formulations followed a Fickian pattern [9].

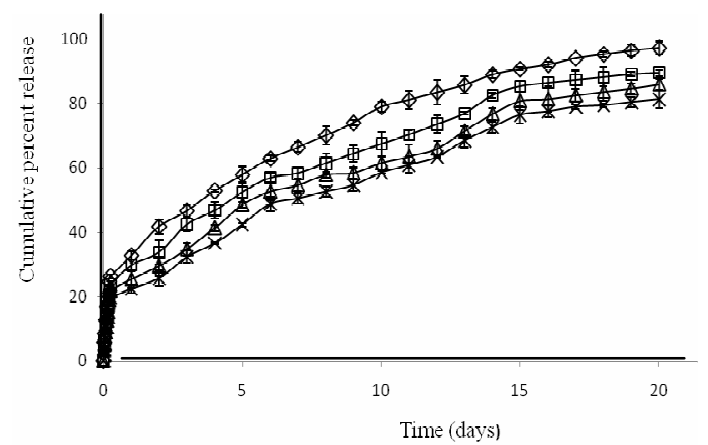

Figure 1: Drug release from silybin nanoparticles $(\diamond=$ NP1, $\square=$ NP2, $\Delta=$ NP3, $x=$ NP4)

\section{Pharmacokinetics of the formulations}

The retention time of the drug, based HPLC studies, was 16.0 and $18.0 \mathrm{~min}$ for the two isomers of silybin. The plasma profiles of the drug after administration of nanoparticles and i.v. solutions are shown in Figure 2. Nanoparticles resulted in higher and more prolonged drug levels than the drug solution. This was reflected by increased area under the curve. The $t_{1 / 2}$, AUC and $V_{d}$ of nanoparticle formulation were higher than for the drug solution, while clearance (CL) was lower than for the free drug (Table 2). All the pharmacokinetic parameters obtained for the nanoparticles were statistically different from those obtained for the drug solution $(p<0.001)$, except Cmax.

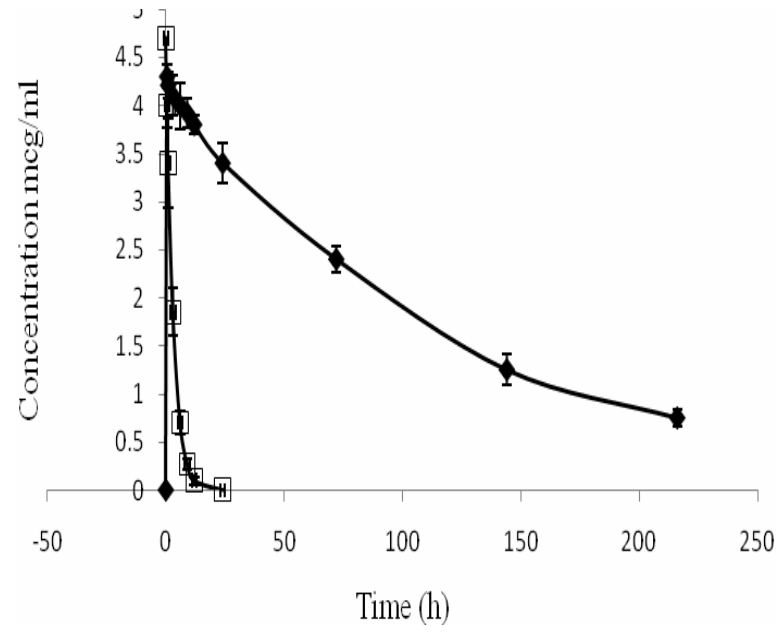

Figure 2: Plasma concentration-time curve of silybin following i.v. administration to rats $(\bullet=$ nanoparticles, $\square=$ solution)

\section{Hepatoprotective activity}

Table 3 shows hepatoprotective activity data. The administration of $\mathrm{CCl}_{4}$ to the animals resulted in a marked increase in SGPT and SGOT activities, indicating increased toxicity, but this was mitigated in the animals treated with silybin formulations. The reduction in toxicity was statistically significant at $p<0.001$ for both sylibinin formulations and sylibin solution.

Table 1: Particle size, charge, PDI and encapsulation efficiency of silybin nanoparticles

\begin{tabular}{lcccc}
\hline Formulation & $\begin{array}{c}\text { Particle size } \\
\text { (mean } \pm \text { SD, } \mathbf{n m})\end{array}$ & $\begin{array}{c}\text { Polydispersity } \\
\text { Index(PDI) }\end{array}$ & $\begin{array}{c}\text { Zeta potential } \\
\text { (mean } \pm \text { SD) }\end{array}$ & $\begin{array}{c}\text { Encapsulation } \\
\text { efficiency (\%) }\end{array}$ \\
\hline $\mathrm{NP}_{1}$ & $135 \pm 3$ & 0.13 & $-31 \pm 3$ & 91.0 \\
$\mathrm{NP}_{2}$ & $220 \pm 2$ & 0.14 & $-36 \pm 3$ & 92.6 \\
$\mathrm{NP}_{3}$ & $310 \pm 2$ & 0.20 & $-34 \pm 3$ & 94.9 \\
$\mathrm{NP}_{4}$ & $426 \pm 1$ & 0.35 & $-37 \pm 3$ & 95.3 \\
\hline
\end{tabular}


Table 2: Pharmacokinetic parameters for silybin formulations administered i.v. (mean $\pm S D, n=3$ )

\begin{tabular}{cll}
\hline Parameter & $\begin{array}{l}\text { Silybin } \\
\text { solution } \\
\text { by i.v. bolus }\end{array}$ & $\begin{array}{l}\text { Silybin } \\
\text { nanoparticles } \\
\text { (NP1) }\end{array}$ \\
\hline $\mathrm{C}_{\max }(\mu \mathrm{g} / \mathrm{ml})$ & $4.7 \pm 0.2$ & $4.3 \pm 0.1$ \\
$\mathrm{Ke}\left(\mathrm{h}^{-1}\right)$ & $0.316 \pm 0.009$ & $0.00791 \pm 0.00016^{* * *}$ \\
$\mathrm{t}_{1 / 2}(\mathrm{~h})$ & $2.19 \pm 0.10$ & $87.6 \pm 1.3^{\star * *}$ \\
$\mathrm{Vd}(\mathrm{L})$ & $0.893 \pm 0.04$ & $0.976 \pm 0.007^{\star * *}$ \\
$\mathrm{Clearance}(\mathrm{L} / \mathrm{h})$ & $0.282 \pm 0.001$ & $0.00715 \pm 0.00012^{* * *}$ \\
AUC ${ }_{0-\infty}(\mu \mathrm{g} . \mathrm{h} / \mathrm{ml})$ & $15.42 \pm 0.24$ & $600.8 \pm 0.8^{* * *}$ \\
${ }^{* * *} p<0.001,{ }^{* *} p<0.01,{ }^{*} p<0.05$, compared with silybin i.v. \\
solution.
\end{tabular}

Table 3: Effect of silybin formulations on enzyme levels in rats with carbon tetrachloride $\left(\mathrm{CCl}_{4}\right)$-induced hepatotoxicity (mean $\pm S D, n=6$ )

\begin{tabular}{|c|c|c|c|c|}
\hline $\begin{array}{l}\text { Treatme } \\
\text { nt } \\
\text { Group }\end{array}$ & $\begin{array}{c}\text { Initial } \\
\text { body } \\
\text { weight (g) }\end{array}$ & $\begin{array}{c}\text { Body } \\
\text { weight } \\
\text { after } 9 \\
\text { days }(\mathrm{g}) \\
\end{array}$ & $\begin{array}{l}\text { SGPT(U } \\
\text { /L) }\end{array}$ & $\begin{array}{c}\text { SGOT( } \\
\text { U/L) }\end{array}$ \\
\hline Control & $160 \pm 10$ & $180 \pm 15$ & $\begin{array}{l}11.1 \pm 2 . \\
3\end{array}$ & $\begin{array}{c}33.3 \pm 2 . \\
9\end{array}$ \\
\hline $\mathrm{CCl}_{4}$ & $165 \pm 15$ & $148 \pm 10$ & $\begin{array}{l}77.8 \pm 3 . \\
7\end{array}$ & $\begin{array}{c}90.0 \pm 3 \\
9\end{array}$ \\
\hline $\begin{array}{l}\text { Silybin } \\
\text { solution }\end{array}$ & $170 \pm 5$ & $180 \pm 5^{\star \star *}$ & $\begin{array}{l}44.4 \pm 4 . \\
6^{\star * \star}\end{array}$ & $\begin{array}{c}63.3 \pm 2 . \\
8^{* * *}\end{array}$ \\
\hline $\begin{array}{l}\text { Drug } \\
\text { nanoparti } \\
\text { cles }\end{array}$ & $170 \pm 6$ & $\underset{\star \star \star}{196 \pm 15^{\star}}$ & $\begin{array}{l}18.4 \pm 3 \\
2^{\star \star \star}\end{array}$ & $\begin{array}{c}47.6 \pm 4 . \\
8^{* \star \star}\end{array}$ \\
\hline $\begin{array}{l}\text { Placebo } \\
\text { nanoparti } \\
\text { cles }\end{array}$ & $165 \pm 6$ & $156 \pm 12$ & $\begin{array}{c}70.4 \pm 2 \\
3^{* \star \star}\end{array}$ & $\begin{array}{c}86.6 \pm 2 . \\
5\end{array}$ \\
\hline
\end{tabular}

However, the drug nanoparticles completely reversed the elevated levels of SGOT and SGPT.

The photomicrographs in Fig 3 display the histological changes in the liver of the animals following administration of the drug-loaded nanoparticles. The histological profile of the control animals showed normal hepatic architecture with distinct hepatic cells, wellpresented cytoplasm sinusoidal spaces and central vein. However, there was disorganization of normal cells with intense centrilobular necrosis following $\quad \mathrm{CCl}_{4} \quad$ intoxication. Moderate accumulation of fatty lobules and cellular necrosis were observed in the animals treated with silybin solution. However, the nanoparticle formulation exhibited strong protection against $\mathrm{CCl}_{4}$-induced liver damage, as evidenced by the presence of normal hepatic cords, well-defined cytoplasm and absence of necrosis. Furthermore, the body weights of the rats which fell significantly after $\mathrm{CCl}_{4}$ treatment were restored to normal following administration of the silybin preparations.

\section{DISCUSSION}

In recent years, various new drug carrier systems in the micro- and nanometer size range have been generated to improve drug delivery. Among them, nanoparticles have certain advantages such as a maximal load of the drug and a long shelf life. Furthermore, their body distribution and permeability in tissues can be controlled by size and surface properties [10]. Previously, we demonstrated the enhanced liver protection of curcumin, curcumin analogues and piperine, using kupffer cell (KC)-targeted nanoparticle and liposomal formulations, and sustained release intraperitoneal microspheres [11-13]. Silybin, a natural antioxidant, has long been used for the treatment of chronic liver diseases. The effectiveness of silybin as a liver disease remedy was discounted by its poor solubility and low bioavailability. It offers low bioavailability due to high first pass metabolism, low $t_{1 / 2}$ and results in patient non compliance due to repeated dosing. Consequently, insufficient concentrations of drugs may accumulate in the target cells. Further, uptake into non-target cells may lead to significant side-effects. Thus we made an attempt to deliver silybin nanoparticles to its target site through bypassing oral route. It can be administered systemically to offer liver protection in a variety of diseases. It can also be targeted to $\mathrm{KC}$ and its delivery into these cells can be enhanced with silybin encapsulated in the particulate systems and further improving the therapy.

To achieve the aims of this study, silybin nanoparticles have been prepared using emulsion solvent evaporation technique. Four different formulations NP1, NP2, NP3 and NP4 were prepared. Particle size and entrapment efficiency of silybin nanoparticles increased with increase in polymer content. This may be due to the availability of more polymer to coat the drug. Increase in polymer content also delayed drug release due to increase in particle size and hence reduced surface area available for drug release. Drug release was highest from NP1 due to because it had the polymer content and this has the effect of retarding drug release as a result of increased particle size and reduced surface area available for drug release. Formulation NP1 was used as the optimized formulation for hepatoprotective test because of its lower particle size and polydispersity index (PDI), and good release profile. This particle size favors uptake into all the liver cells responsible for the formation of liver fibrosis [14]. Higher uptake of particles into KC occurs by passive targeting. 


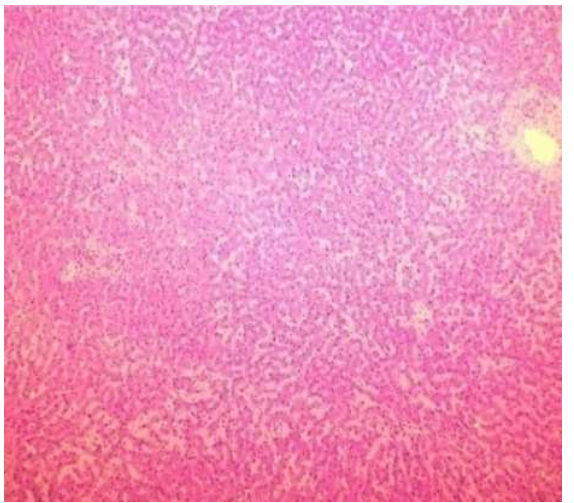

a) Normal liver

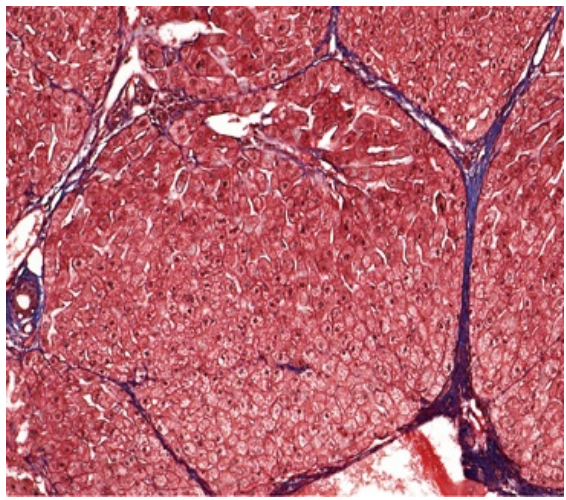

c) Liver treated with silybin solution

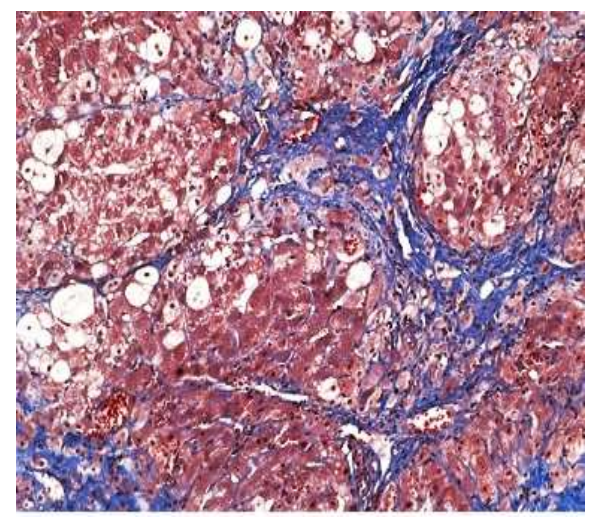

b) $\mathrm{CCl}_{4}$ treated liver

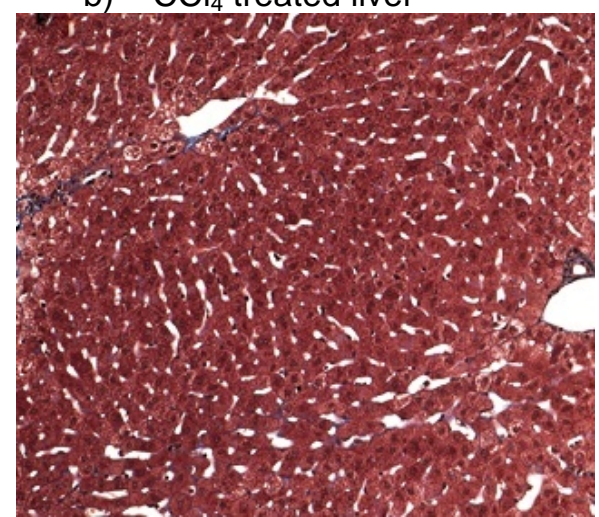

d) Liver treated with Silybin nanoparticles

Figure 3: Histopathological profile of rat liver following nanoparticle administration

NP1 sustained drug release for up to 10 days in vivo and offered better pharmacokinetic properties than the drug in solution form. Elimination rate constant and clearance than for the drug solution. Furthermore, area under the curve (AUC) was several-fold than for the solution. This suggests reduction in drug elimination and metabolism with nanoparticle formulation administration.

Carbon tetrachloride, a known hepatotoxin, is a commonly used model for hepatoprotective drug screening, and the severity of the liver damage is measured by the levels of elevated cytoplasmic enzymes (SGOT and SGPT) in circulation [15]. The hepatocellular damage induced by $\mathrm{CCl}_{4}$ is due to its metabolite, trichloromethyl free radical $\left(\mathrm{CCl}_{3}\right)$ that binds to lipoprotein and leads to peroxidation of the lipids of the endoplasmic reticulum [16] by the action of the mixed function of the cytochrome P450 oxygenase system. This free radical, which is initially relatively unreactive when formed initially, reacts very rapidly with oxygen to yield a highly reactive trichloromethyl peroxy radical $\left(\mathrm{CCl}_{3} \mathrm{OO}\right)$. Both radicals are capable of binding to proteins or abstracting a hydrogen atom from an unsaturated lipid, thus accelerating lipid peroxidation. Expectedly, SGOT and SGPT levels were higher in $\mathrm{CCl}_{4}^{-}$ treated animals due to tissue damage caused by $\mathrm{CCl}_{4}$ which results in the release of the enzymes in to the blood stream. Upon administration, silybin reduced elevated enzyme levels. Reversal of liver toxicity was greater for nanoparticle formulation than for the drug solution form probably because the nanosized particles were instantly taken up by $\mathrm{KC}$ as well as by several other liver cells. As a result, the drug becomes accumulated in the liver cells and is subsequently released at the cellular level to achieve better hepatoprotection.

Overall, the findings of this study suggest that the developed nanoparticle formulation may be useful in the treatment of cirrhosis and fibrosis with silybin. It is likely that these results can be extrapolated to other drugs, suggesting the probability of nanoparticulate passive targeting of drugs to the liver, including for the treatment diseases such as liver cancers.

\section{CONCLUSION}

Silybin nanoparticles can be suitably prepared by emulsion solvent evaporation technique using polycaprolactone as a biodegradable polymer. The particles showed good encapsulation efficiency and sustained drug release both in 
vitro and in vivo. Silybin nanoparticles offer an effective approach for drug targeting of the liver.

\section{REFERENCES}

1. Luster MI, Simeonova PP, Gallucci RM, Bruccoleri A, Blazka ME, Yucesoy B. Role of

inflammation in chemical-induced hepatotoxicity. Toxicol Letters 2001; 120: 317-325.

2. Reinhard S, Remy $M$, Reto $B$. The use of silymarin in treatment of liver disease. Drugs 2001; 61: 2035 2063.

3. Bataller R, Brenner DA. Hepatic stellate cells as a target for the treatment of liver fibrosis. Semin Liver Dis 2001; 21:437-451.

4. Ikeda K, Wang LH, Torres R, Olaso EJ, Klein R, Lovett $D$, Yancopoulos GD, Friedman SL, Lin HC. Discoidin domain receptor 2 (DDR2) interacts with $S R C$ and SHC following activation by collagen I. Hepatology 2000; 32: 315-319.

5. Pradhan SC, Girish C. Hepatoprotective herbal drug Silymarin from experimental pharmacology to clinical medicine. Indian J Med Res 2006; 124: 491-504.

6. Owens III DE, Peppas NA. Opsonization, biodistribution, and pharmacokinetics of polymeric nanoparticles. Int J Pharm 2006; 307: 93-102.

7. CPCSEA guidelines for laboratory animal facility. Indian J Pharmacol. 2003; 35: 257-274.

8. Demers G, Griffin G, De Vroey G, Haywood JR, Zurlo J, Bedard M. Harmonization of Animal Care and Use Guidance. Science 2006; 312: 700-701.
9. Gautam S, Mahaveer S. In-vitro drug release characterization models. Int J Pharm Studies Res 2011; 2(1): 77-84.

10. Stayton PS, Hoffman AS, Murthy N, Lackey C, Cheung C, Tan P, Klumb LA, Chilkoti A, Wilbur FS, Press OW. Molecular engineering of proteins and polymers for targeting and intracellular delivery of therapeutics. J Control Release 2000; 65: 203-220.

11. Bonepally CR, Aukunuru JV, Yellu NR, Vanga MR. Fabrication and Investigations on Hepatoprotective Activity of Sustained Release Biodegradable Piperine Microspheres. Int J Pharm Sci and Nanotech 2008; 1: 87-96.

12. Anuradha CA, Aukunuru J. Preparation, characterization and in vivo evaluation of Bis-demethoxy curcumin analogue (BDMCA) nanoparticles. Trop $J$ Pharm Res 2010; 9: 51-58.

13. Konatham S, Nyathani $H$, Bonepally $C R$, Yeannameneni $P$, Aukunuru J. Liposomal delivery of curcumin to liver. Turk J Pharm Sci 2010; 7: 89-98.

14. Aukunuru J, Bonepally CR. Liver targeting drug delivery. In: Madhusudan Rao Y; Aukunuru J, editors. Advances in Drug Delivery. $1^{\text {st }}$ edn. Hyderabad; Pharma Book Syndicate 2012; pp 267-315.

15. Reitman S, Frankel AS. A colorimetric method for the determination of serum glutamyl oxalacetic acid glutamyl pyruvate transaminase. Am J Clin Pathol 1957; 28: 56-58.

16. Recknagael RO. Carbon tetrachloride hepatotoxicity. Pharmacol Rev 1967; 19: 145-208. 